\title{
Engraftment, Fate, and Function of HoxB8-Conditional Neutrophil Progenitors in the Unconditioned Murine Host
}

OPEN ACCESS

Edited by:

Zhichao Fan,

UCONN Health, United States

Reviewed by:

Rongrong Liu,

Northwestern University,

United States

Yi Zhang,

Fudan University, China

${ }^{*}$ Correspondence:

Craig T. Lefort

craig_lefort@brown.edu

${ }^{\dagger}$ These authors have contributed equally to this work

Specialty section:

This article was submitted to Cell Adhesion and Migration, a section of the journal Frontiers in Cell and Developmental Biology

Received: 21 December 2021 Accepted: 05 January 2022 Published: 20 January 2022

Citation:

Cohen JT, Danise M, Hinman KD, Neumann BM, Johnson R, Wilson ZS,

Chorzalska A, Dubielecka PM and Lefort CT (2022) Engraftment, Fate, and Function of HoxB8-Conditional Neutrophil Progenitors in the Unconditioned Murine Host. Front. Cell Dev. Biol. 10:840894. doi: 10.3389/fcell.2022.840894
Joshua T. Cohen ${ }^{1 \dagger}$, Michael Danise ${ }^{1 \dagger}$, Kristina D. Hinman ${ }^{1,2}$, Brittany M. Neumann ${ }^{1}$, Renita Johnson ${ }^{1}$, Zachary S. Wilson ${ }^{1,2}$, Anna Chorzalska ${ }^{3}$, Patrycja M. Dubielecka ${ }^{3}$ and Craig T. Lefort ${ }^{1 *}$

${ }^{1}$ Division of Surgical Research, Department of Surgery, Rhode Island Hospital, Providence, RI, United States, ${ }^{2}$ Graduate Program in Pathobiology, Brown University, Providence, RI, United States, ${ }^{3}$ Division of Hematology/Oncology, Rhode Island Hospital, Providence, Rl, United States

The development and use of murine myeloid progenitor cell lines that are conditionally immortalized through expression of HoxB8 has provided a valuable tool for studies of neutrophil biology. Recent work has extended the utility of HoxB8-conditional progenitors to the in vivo setting via their transplantation into irradiated mice. Here, we describe the isolation of HoxB8-conditional progenitor cell lines that are unique in their ability to engraft in the naive host in the absence of conditioning of the hematopoietic niche. Our results indicate that HoxB8-conditional progenitors engraft in a $\beta 1$ integrin-dependent manner and transiently generate donor-derived mature neutrophils. Furthermore, we show that neutrophils derived in vivo from transplanted HoxB8-conditional progenitors are mobilized to the periphery and recruited to sites of inflammation in a manner that depends on the $\mathrm{C}-\mathrm{X}-\mathrm{C}$ chemokine receptor 2 and $\beta 2$ integrins, the same mechanisms that have been described for recruitment of endogenous primary neutrophils. Together, our studies advance the understanding of HoxB8-conditional neutrophil progenitors and describe an innovative tool that, by virtue of its ability to engraft in the naïve host, will facilitate mechanistic in vivo experimentation on neutrophils.

Keywords: neutrophils, hematopoietic stem and progenitor cell (HSPC), engraftment, granulopoeisis, cell therapeutics, leukocyte recruitment

\section{INTRODUCTION}

As mediators of the acute inflammatory response, neutrophils play essential roles in host defense against bacterial and fungal infection (Ley et al., 2018). Neutrophil development into their mature effector state primarily occurs in the bone marrow. Neutrophils are abundant and short-lived, a combination that requires the expenditure of a great amount of energy and resources to produce around a billion mature neutrophils per kilogram human body mass per day under homeostasis (Kubes, 2018). The rationale for the host's investment in the neutrophil is underscored by the manifestation of recurrent and severe infections in individuals with neutropenia or neutrophil dysfunction (Ley et al., 2018).

While primary neutrophils are easily obtained from blood or tissue samples, they are nonproliferative and have a short lifespan. Moreover, common techniques for introducing genetic 
material to cells result in artefactual activation of primary neutrophils. Thus, even in the current age of facile genetic engineering, mechanistic investigation of neutrophil biology remains a challenge. For decades, neutrophil biologists have relied on imperfect "neutrophil-like" cell lines that fail to fully recapitulate the breadth of neutrophil functionality and may differ from primary neutrophils mechanistically in those functions. Such models include human HL-60 cells (Collins et al., 1978) and murine MPRO (Lawson et al., 1998) and 32D clone 3 (Valtieri et al., 1987) cell lines. The limitations of these neutrophil-like cell models have been comprehensively reviewed (Gupta et al., 2014; Blanter et al., 2021).

More recently, an improved approach was described for deriving neutrophils from murine myeloid progenitors that are conditionally immortalized through controlled expression of HoxB8 (Wang et al., 2006). Expression of HoxB8, a member of the homeobox family of transcription factors, blocks the terminal differentiation of progenitors into monocytes or granulocytes (Krishnaraju et al., 1997; Knoepfler et al., 2001). Neutrophils differentiated in vitro from HoxB8-conditional murine myeloid progenitors have been extensively characterized and have shown much promise in replicating the biological functions displayed ex vivo by isolated murine neutrophils (McDonald et al., 2011; Weiss et al., 2018; Zehrer et al., 2018; Chu et al., 2019; Saul et al., 2019).

Recent studies have further extended the utilization of HoxB8conditional progenitors as an experimental tool for in vivo studies via their transplantation into mice. Among the wide range of genetic disorders that impact neutrophil development or function, there are more than a dozen different genes whose mutation underlies severe congenital neutropenia as component of the phenotype (Ley et al., 2018). Deficiency of glucose-6phosphatase- $\beta$ (G6PC3), an enzyme involved in glucose metabolism, results in neutropenia in humans (Boztug et al., 2009) and mice (Cheung et al., 2007). Utilizing HoxB8conditional progenitors derived from mice deficient in G6PC3, the neutropenic phenotype was replicated and enabled the investigators to gain mechanistic insight into the role of G6PC3 in neutrophil development (Gautam et al., 2013). In those studies, the HoxB8-conditional progenitor system permitted the exogenous expression of the anti-apoptotic protein $\mathrm{Bcl}-\mathrm{X}_{\mathrm{L}}$ to disentangle the defects in neutrophil progenitor survival from defects in differentiation (Gautam et al., 2013). In another study that employed a mouse model of arthritis, neutrophils derived in vivo from HoxB8-conditional progenitors that were adoptively transferred into conditioned mice could be recruited to the inflamed joint (Orosz et al., 2021). In the absence of endogenous host neutrophils, mice that received HoxB8-conditional progenitors exhibited ankle swelling and a clinical score that mirrored that of control mice with intact endogenous neutrophils (Orosz et al., 2021).

There is accumulating evidence that HoxB8-conditional progenitors could be a tool for neutrophil biologists to perform mechanistic and disease model studies in vivo on primary-like neutrophils. However, the most significant limitation and drawback to the work described above is that in each study recipient mice needed to be conditioned with irradiation to allow repopulation of the hematopoietic compartment with HoxB8-conditional progenitors. Myeloablative conditioning results in irradiation- or chemicalinduced toxicity and inflammation that can significantly impact mouse disease models and confound mechanistic studies (Xun et al., 1994; Duran-Struuck and Dysko, 2009). Orosz and others recently demonstrated the absolute requirement for lethally irradiating recipient mice to achieve engraftment of their HoxB8-conditional progenitors (Orosz et al., 2021). Here, we describe the novel discovery of an independently derived HoxB8conditional progenitor cell line that is able to robustly engraft in naïve recipient mice, in the absence of conditioning the hematopoietic niche. Our results also indicate that neutrophils generated in vivo from transplanted HoxB8-conditional progenitors are functionally equivalent to endogenous primary neutrophils, in terms of their trafficking and effector activities. Together, these results address a key challenge in the field in developing a facile and tractable tool for mechanistic studies of neutrophil biology.

\section{MATERIALS AND METHODS \\ HoxB8 Conditionally-Immortalized Progenitors}

All three of the independent derivations of HoxB8-conditional progenitor cell lines analyzed were derived using the same protocol. As described previously (Cohen et al., 2021), a lentivirus-based construct with a tamoxifen-inducible Hoxb8 oncogene (Salmanidis et al., 2013) (a gift from Paul Ekert, Murdoch Children's Research Institute) with either a puromycin or hygromycin resistance cassette was used to stably express HoxB8 in bone marrow hematopoietic stem and progenitor cells (HSPCs) isolated from C57BL/6 J mice using a kit (Stemcell Technologies). HSPCs were cultured with recombinant murine stem cell factor (SCF) and interleukin-3 (IL-3) for 2 days, exactly as described (Wang et al., 2006), and then subjected to spin-infection (2000 RPM, $60 \mathrm{~min}, 30^{\circ} \mathrm{C}$ ) with lentivirus in a 24 well plate. Cell culture media consisted of Opti-MEM containing GlutaMax (ThermoFisher), $30 \mu \mathrm{M}$ beta-mercaptoethanol (Sigma-Aldrich), $\quad 10 \%$ fetal bovine serum (Gemini Bioproducts), penicillin/streptomycin (Gibco), and nonessential amino acids (Gibco). To establish conditionallyimmortalized progenitor lines, cells were cultured in media supplemented with $50 \mathrm{ng} / \mathrm{ml}$ SCF (BioLegend), $100 \mathrm{nM} \mathrm{Z-4-}$ hydroxytamoxifen (Tocris), and either puromycin (SigmaAldrich) or hygromycin (Sigma-Aldrich) for up to 2 weeks. In some cases, we used conditioned media from $\mathrm{CHO}$ cells that secrete recombinant murine SCF (a gift from Patrice Dubreuil, Centre de Recherche en Cancérologie de Marseille).

\section{Genetic Disruption Using CRISPR/Cas9}

To generate gene knockout cell lines, we used pLentiCRISPR v2 vector, a gift from Feng Zhang (Addgene plasmid \#52961). The following 20-nucleotide single-guide RNA (sgRNA) targeting sequences were inserted into pLentiCRSIPR v2: CGGAAG CGAGGTGCAGACCG (Itga4), AGTGACATAGAGAATCCC 
AG (Itgb1), AATGTCATCGCGGCGCTCAC (Cxcr2); Tln1 and Itgb2 targeting have been previously described (Wilson et al., 2017). Lentivirus was produced using a HEK293T Lenti-X cell line (Takara Bio). HoxB8-conditional progenitors were transduced with lentivirus in the presence of $5 \mu \mathrm{g} / \mathrm{ml}$ polybrene (Sigma-Aldrich) in a 24 -well plate subjected to centrifugation at $800 \times \mathrm{g}$ for $60 \mathrm{~min}$.

\section{Animals and HoxB8-Conditional Progenitor Transplantation}

These studies were approved by the Lifespan Animal Welfare Committee (Approval \# 5017-19, Office of Laboratory Animal Welfare Assurance \#A3922-01) and were conducted in accordance with Public Health Service guidelines for animal care and use. Experiments used 8-14-week old C57BL/6 or CD45.1 (B6. SJL-PtprcaPepcb/BoyJ) mice (The Jackson Laboratory). Water and standard chow were available ad libitum. For cell transplantation, CD45.1 mice were anesthetized with $3 \%$ isoflurane and received $1 \times 10^{8}$ (unless otherwise indicated) HoxB8-conditional progenitors via retroorbital i. v. injection.

\section{Antibodies}

Unless otherwise specified, the following antibodies were from BioLegend: anti-Ly6G (1A8), anti-CD45.1 (A20), anti-CD45.2 (104), anti-cKit (ACK2), anti-CXCR4 (FAB21651, R\&D Systems), anti-CD11a (M17/4), anti-CD11b (M1/70), antiCD18 (M18/2), anti-CD29 (HMB1-1), anti-CD49d (R1-2), anti-CD49e (5H10-27), anti- $\beta 7$ integrin (FIB27), anti-CD44 (IM7), anti-L-selectin (MEL-14), anti-CD16/32 (93), anti-Ly6C (HK1.4), anti-CXCR2 (SA044G4), anti-CD101 (Moushi101, Invitrogen), anti-Fc $\gamma$ RIV (9E9), anti-CD162 2PH1, BD Biosciences).

\section{Analyses of Blood and Bone Marrow}

At the indicated time after transplantation of HoxB8-conditional progenitors, blood samples were collected by saphenous venipuncture in K3-EDTA tubes (Sarstedt). Blood samples were subjected to erythrocyte lysis with ammonium chloride (BioLegend). For harvesting bone marrow, mice were euthanized and then femurs and tibias were flushed with ice cold PBS containing $5 \mathrm{mM}$ EDTA and 0.5\% BSA (PEB buffer) through 70- $\mu$ m nylon filters. Samples in PEB buffer were labeled with antibodies as indicated, washed extensively with PEB buffer, and then analyzed by flow cytometry using a MACSQuant Analyzer 10. Data analysis was performed using FlowJo software (BD Biosciences).

\section{Mouse Model of Thioglycollate-Induced Peritonitis}

On day 7 post-transplant, an initial blood sample was collected from CD45.1 recipient mice. Mice then received intraperitoneal injection of $1 \mathrm{ml} 4 \%$ thioglycollate broth (Sigma-Aldrich). Approximately $30 \mathrm{~min}$ prior to the end point, a "post-thio" blood sample was collected. At $6 \mathrm{~h}$ after injection of thioglycollate, mice were euthanized and the peritoneal lavage was collected by flushing the peritoneal cavity with $5 \mathrm{ml}$ ice cold PEB buffer. Blood and peritoneal lavage samples were labeled with antibodies against CD45.1, CD45.2, Ly6G, CD18 and/or CXCR2 as indicated, and then analyzed by flow cytometry as described above.

\section{Phagocytosis Assay}

Bone marrow was harvested as described above and split into samples of approximately $2.5 \times 10^{5}$ cells each in Hanks' balanced salt solution containing $\mathrm{Ca}^{2+} / \mathrm{Mg}^{2+}$ (HBSS) and $10 \%$ FBS. Some samples were pretreated with $10 \mu \mathrm{g} / \mathrm{ml}$ cytochalasin $\mathrm{D}$ prior to adding $10 \mu \mathrm{g} / \mathrm{mL} S$. aureus bioparticles conjugated with a pHrodo-Green fluorescent probe (ThermoFisher Scientific). After allowing phagocytosis by incubating samples at $37^{\circ} \mathrm{C}$ for $60 \mathrm{~min}$, cells were washed twice with ice cold PEB buffer. Samples were labeled with antibodies against CD45.2 and Ly6G before analysis by flow cytometry as described above.

\section{S. aureus Intracellular Killing Assay}

A GFP-expressing strain of S. aureus, USA300-sGFP (a gift from Alexander Horswill, University of Colorado Denver), was grown overnight in tryptic soy broth, then diluted 1:10 in fresh broth and grown to log phase. After extensive washing in PBS, the colony forming unit (CFU) density of USA300-sGFP was quantified by measuring its optical density at $600 \mathrm{~nm}$ (OD600) on a spectrophotometer. Anti-coagulated blood samples from recipient mice were subjected to lysis of erythrocytes with ammonium chloride (BioLegend) and then labeled at room temperature with antibodies against Ly6G and CD45.2. After washing, samples were resuspended in HBSS containing $10 \%$ FBS. Vehicle control or USA300-sGFP $\left(6 \times 10^{5} \mathrm{CFU} /\right.$ sample $)$ was added to samples and they were incubated at $37^{\circ} \mathrm{C}$ for $15 \mathrm{~min}$ to allow $S$. aureus uptake. To remove remaining extracellular $S$. aureus, samples were washed in the presence of $10 \mu \mathrm{g} / \mathrm{mL}$ lysostaphin, pelleted, and resuspended in HBSS containing $10 \%$ FBS. A portion of the sample was transferred to ice for analysis by flow cytometry as the initial $0 \mathrm{~min}$ time point. The remaining sample was incubated at $37^{\circ} \mathrm{C}$, then transferred to ice for analysis by flow cytometry as described above.

\section{Respiratory Burst Assay}

Anti-coagulated blood samples from recipient mice were diluted 10 -fold in HBSS containing $\mathrm{Ca}^{2+} / \mathrm{Mg}^{2+}$. Samples were then loaded for $15 \mathrm{~min}$ with $2.5 \mu \mathrm{g} / \mathrm{ml}$ dihydrorhodamine 123 (DHR123; Cayman Chemical), a probe that fluoresces upon exposure to reactive oxygen intermediates. After DHR123 loading, diphenyleneiodonium (DPI; Sigma Aldrich) was added to the appropriate samples at a concentration of $10 \mu \mathrm{M}$ just prior to stimulation of samples with either heat-killed $S$. aureus (HKSA) or $100 \mathrm{ng} / \mathrm{ml}$ phorbol 12-myristate 13-acetate (PMA; Sigma-Aldrich). Samples were stimulated for either 15 min (PMA) or $30 \mathrm{~min}$ (HKSA). At the end of stimulation, samples were centrifuged to pellet cells, subjected to red blood cell lysis as described above, and then stained on ice with antibodies against CD45.2, CD45.1, and Ly6G. Samples were analyzed by flow cytometry as described above. 


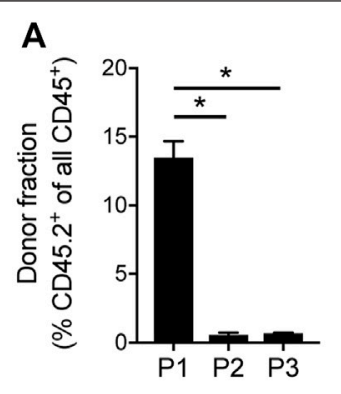

C

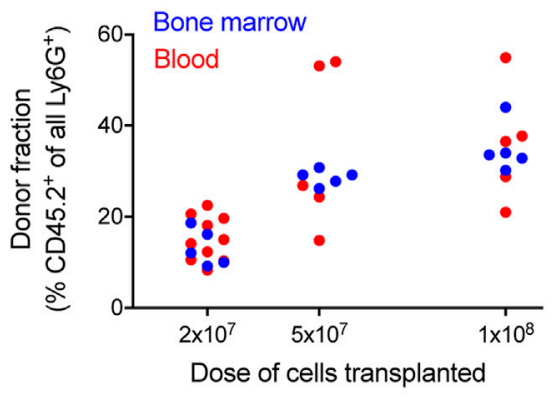

B

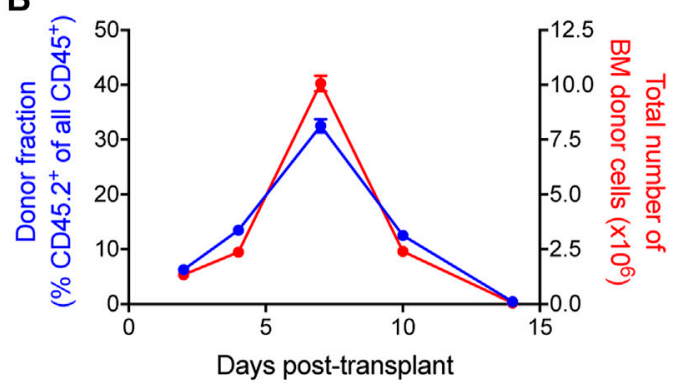

D

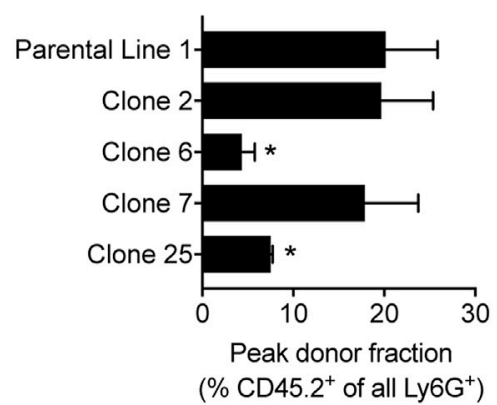

FIGURE 1 | Quantification of HoxB8-conditional progenitor engraftment in unconditioned mice. (A) CD45.1 mice received transplantation of $1 \times 10^{8}$ progenitors of one of the three indicated HoxB8-conditional progenitor cell lines that were independently established as described in the Methods. The fraction of all CD45 ${ }^{+}$bone marrow cells that were derived from transplanted HoxB8-conditional progenitors $\left(C D 45.2^{+}\right)$in bone marrow of recipient mice at 5 days after transplantation. HoxB8conditional progenitor line 1, line 2, and line 3 are indicated here as P1, P2, and P3, respectively. Data were analyzed using one-way ANOVA. ${ }^{*} P<0.05$. (B) CD45.1 mice received transplantation of $1 \times 10^{8} \mathrm{HoxB} 8$-conditional progenitors (line 1) to perform a time course of both the fraction (blue, left $y$-axis) and absolute number (red, right $y$-axis) of donor-derived cells in the bone marrow. (C) The donor-derived (CD45.2+) fraction of all mature Ly6G high neutrophils in the bone marrow CD45.1 mice that received varying doses of HoxB8-conditional progenitors, measured at 7 days post-transplant. (D) Evaluation of the maximum fraction of donor-derived (CD45.2 ${ }^{+}$) neutrophils in the peripheral blood of CD45.1 mice that received transplant of $3 \times 10^{7}$ cells of either the parental HoxB8-conditional progenitor cell line 1 or one of the single-cell clones derived from that cell line. Data were analyzed using one-way ANOVA. ${ }^{*} p<0.05$, compared to parental line 1.

\section{Statistical Analyses}

All statistical analyses were performed using Prism 8 (GraphPad). Data throughout are presented as the group mean and standard deviation. One-way or two-way analysis of variance (ANOVA) was used to compare the differences between group means and post-hoc analysis was performed using a Bonferroni multiple comparison test. A paired or unpaired (as appropriate) Student's $t$-test was used for statistical comparisons limited to two groups.

\section{RESULTS}

\section{Engraftment of HoxB8-Conditional Progenitors}

Since the approach for conditionally immortalizing murine myeloid progenitors by exogenous expression of HoxB8 was initially described (Wang et al., 2006), others have shown that such progenitor cell lines can be transplanted into irradiated recipient mice and subsequently generate mature, terminally differentiated myeloid leukocytes in the in vivo environment (Gautam et al., 2013; Redecke et al., 2013; Orosz et al., 2021). Recently, we reported that a HoxB8-conditional progenitor cell line that we derived is able to produce substantial numbers of donor graft-derived neutrophils in mice that were not subjected to any conditioning of the hematopoietic niche (Cohen et al., 2021). To understand if the engraftment of HoxB8-conditional progenitors in naïve, unconditioned mice was a unique feature of this particular progenitor cell line, we compared its engraftment to two additional and distinct lines of HoxB8-conditional myeloid progenitors using congenic CD45.1 recipient mice. Each of these three cell lines tested were independently generated using the same protocol, by lentiviral delivery of a tamoxifen-inducible Hoxb8 transgene to hematopoietic stem and progenitor cells (HSPCs) isolated from the bone marrow of $\mathrm{C} 57 \mathrm{BL} / 6 \mathrm{~J}$ mice $\left(\mathrm{CD} 45.2^{+}\right)$. To determine whether three distinct HoxB8conditional progenitor lines would home to and take up residence in the bone marrow, we transplanted them separately in CD45.1 mice that had not received any prior conditioning regimen. For two of the independentlyestablished progenitor lines, line 2 (P2) and line 3 (P3), we observed very few donor-derived cells present in the recipient mouse bone marrow at 4 days after transplant (Figure 1A). This was in contrast to the robust engraftment of progenitor line 1 (P1), as measured by the fraction of CD $45.2^{+}$cells in bone marrow (among all $\mathrm{CD}_{4} 5^{+}$cells) that were derived from transplanted donor progenitors (Figure 1A). These results 
indicate that HoxB8-conditional progenitor line 1 has a unique capacity for hematopoietic-intact engraftment.

To further characterize the engraftment of HoxB8-conditional progenitor cell line 1 , we used CD45.1 recipient mice and performed a kinetic analysis of donor-derived CD45.2 $2^{+}$cells in the bone marrow. In this experiment, CD45.1 received $1 \times 10^{8}$ HoxB8-conditional progenitors on day 0 . The fraction of all bone marrow $\mathrm{CD}_{4} 5^{+}$cells that are derived from transplanted donor cells peaks around day 7 after transplant and are nearly undetectable by day 14 (Figure 1B), following kinetics similar to those observed by others in irradiated recipient mice (Orosz et al., 2021). The absolute number of CD $45.2^{+}$donor-derived cells in the bone marrow follows a similar trajectory (Figure 1B), suggesting that a relatively small fraction of transplanted HoxB8conditional progenitors initially engraft and that it is their proliferation within the hematopoietic niche that drives the substantial donor chimerism that develops over the first week following transplant. Furthermore, we observed a dosedependence between the number of transplanted progenitors and the fraction of neutrophils in the bone marrow and peripheral blood that were donor-derived on day 7 after transplant (Figure 1C). At longer time points of 45 days and 6 months, we did not detect any donor-derived cells in the bone marrow and there was not a detectable long-term impact on the host stem/progenitor cell composition of the bone marrow (Supplementary Figure S1). These data suggest that once exogenous tamoxifen-driven HoxB8 expression is turned off, donor progenitors enter a terminal differentiation program and their existence is then solely determined by the mature neutrophil lifespan.

The derivation of HoxB8-conditional progenitors from bone marrow HSPCs is likely to produce a heterogeneous mix of conditionally-immortalized myeloid progenitor states. To further understand whether the disparate engraftment among the three HoxB8-immortalized cell lines was due to the presence of a subpopulation with an intrinsic ability to engraft in the bone marrow niche, we generated single-cell clones of progenitor line 1 and then analyzed the engraftment of four distinct clonal progenitor lines that were transplanted into naive CD45.1 recipient mice. We analyzed peripheral blood longitudinally to assess the peak fraction of donor-derived neutrophils in the periphery among the different clonal lines tested, as there was some variability between progenitor clones in their kinetics of engraftment and differentiation. In addition, we utilized a transplantation dose of $3 \times 10^{7}$ HoxB8-conditional progenitors in order to better resolve potential differences between progenitor line 1 and the clonal progenitor lines. Across four different singlecell clones, we observed a range in the peak fraction of peripheral blood neutrophils that were derived from transplanted donor progenitors (Figure 1D). Clones 6 and 25 exhibited a reduced peak frequency of donor-derived neutrophils in the periphery compared to the parental line 1, whereas the donor fraction for clones 2 and 7 was comparable to that of the parental progenitor line 1 (Figure 1D). These data suggest the presence of a subset of highly-engraftable progenitors within the parental HoxB8conditional progenitor line 1 . As these analyses involved serial sampling of the peripheral blood, we accrued a substantial number of data points to be able to assess whether neutrophils generated from transplanted donor progenitors displace those generated via endogenous host granulopoiesis. There was no detectable relationship between the host-derived neutrophil count in the blood and the fraction of all neutrophils that were derived from the donor (Supplementary Figure S1).

To gain insight into potential cell surface receptors that may regulate engraftment or define a subset of highly-engrafting neutrophil progenitors, we performed flow cytometry analyses of HoxB8-conditional progenitor lines and single-cell clones to evaluate expression of cKit; C-X-C chemokine receptor 4 (CXCR4); integrin subunits CD11a, CD11b, and CD29; CD44, L-selectin, CD16/32, CD101, Ly6C, and Ly6G. Most of these receptors and markers exhibited homogeneous expression levels within each line (Supplementary Figure S2) and many had similar expression across the different progenitor cell lines analyzed (Figure 2). All progenitor lines lacked expression of Ly6G and CD101 (Supplementary Figure S2). Notable differences between progenitor lines include higher cKit expression for the poorly engrafting line 2 and disparate expression levels of several receptors/markers (CD11a, CD29) on progenitor line 3 (Figure 2). There was a prominent Ly6 $\mathrm{C}^{\text {high }}$ population within line 1 , line 3 , and all of the clonal progenitor lines derived from progenitor line 1 (Figure 2). Compared to line 1 , line 2 had a reduced fraction of Ly $6 C^{\text {high }}$ progenitors while line 3 exhibited a near $100 \%$ fraction of the Ly $6 \mathrm{C}^{\text {high }}$ population (Figure 2). However, the frequency of the Ly6C $\mathrm{C}^{\text {high }}$ subpopulation exhibited no apparent correlation with the intrinsic engraftment capacity of the respective HoxB8conditional progenitor cell lines. Together, these data suggest that conditional HoxB8 expression in myeloid progenitors may lead to the immortalization of several different types of progenitors.

\section{Mechanisms of HoxB8-Conditional Progenitor Engraftment}

Prior to taking up residence in the hematopoietic niche, transplanted HSPCs must first traffic from the circulation to the bone marrow. Broadly, HSCs (hematopoietic stem cells) and HPCs (hematopoietic progenitor cells) transplanted into conditioned recipients undergo engraftment that depends on the integrin VLA-4 (Papayannopoulou et al., 1995; Potocnik et al., 2000; Qian et al., 2007; Rettig et al., 2012). To determine whether VLA-4 plays a role in the engraftment of HoxB8-conditional progenitors, we used CRISPR/Cas9 to generate progenitor cell lines deficient in either Itga4 or Itgb1 that encode the $\alpha 4$ and $\beta 1$ subunits of VLA-4, respectively (Supplementary Figure S3). Congenic CD45.1 mice received a 1:1 mix of wild-type and either Itga $^{-/-}$or $\operatorname{Itgb1}^{-/-}$HoxB8conditional progenitors. At 5 days post-transplant, we measured the relative fraction of wild-type and knockout CD $45.2^{+}$donor-derived cells in the bone marrow (Figure 3A). Interestingly, in the respective recipient mice, the abundance of Itga $^{-1-}$ donor cells was similar to that of wild-type donor cells (Figure 3A). In contrast, Itgb1 ${ }^{-/-}$HoxB8-conditional progenitors exhibited a level of engraftment that was significantly less than 

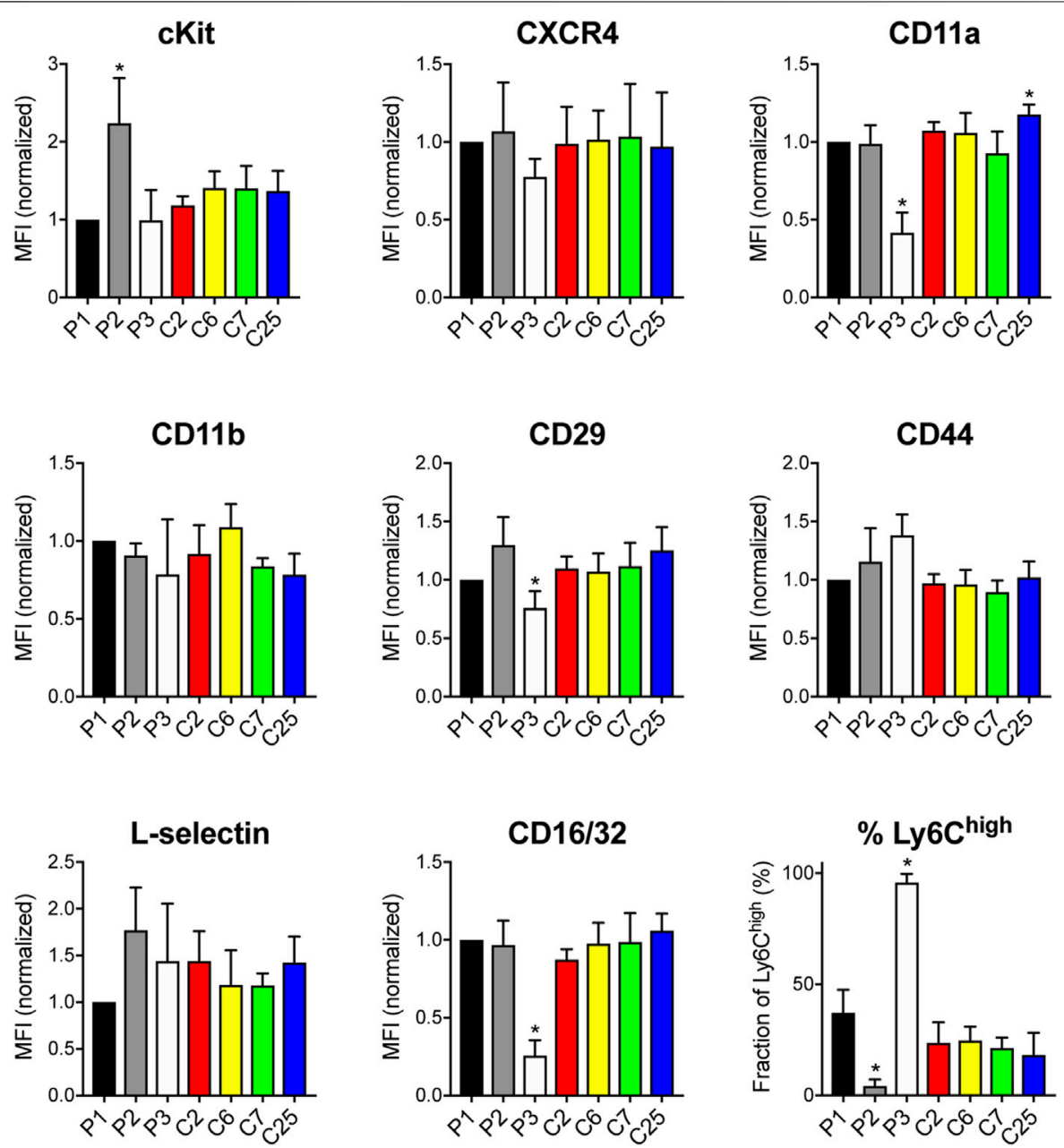

FIGURE 2 | Receptor and surface marker expression on HoxB8-conditional progenitors. Flow cytometry analyses was performed to quantify the cell surface expression of the indicated receptors and markers on the three main HoxB8-conditional progenitor cell lines; line 1 (P1), line 2 (P2), line 3 (P3); and the P1-derived singlecell clonal lines designated clone 2 (C2), clone 6 (C6), clone 7 (C7), and clone 25 (C25). Data were analyzed using one-way ANOVA. ${ }^{*} p<0.05$, compared to P1.

that of wild-type (Figure 3A). These data indicate that VLA-4 is not essential for the engraftment of HoxB8-conditional progenitors, but that another $\beta 1$ integrin subtype plays a role in this process. Flow cytometry analyses of the $\operatorname{Itga~}^{-1-}$ progenitor cell line indicated that CD29 ( $\beta 1$ integrin subunit) remains expressed at the cell surface (Supplementary Figure S3), indicating that other $\beta 1$ integrins are expressed. Wild-type, but not $\operatorname{Itgb1} 1^{-1-}$, progenitors expressed CD49e and CD49f that pair with $\mathrm{CD} 29$ to form $\alpha 5 \beta 1$ and $\alpha 6 \beta 1$ integrins, respectively (Supplementary Figure S3). Wild-type, but not $\operatorname{Itga4}^{-/-}$, progenitors also expressed a $4 \beta 7$ integrins (Supplementary Figure S3).

Extravasation of some leukocyte subsets from blood vessels is dependent on $\beta 2$ integrins and a recent study suggests that $\beta 2$ integrins contribute to transplanted HSPC homing (Mei et al., 2020). In contrast to our observations of the reduced engraftment of $\beta 1$ integrin-deficient HoxB8-conditional progenitors, Itgb2 $2^{-1-}$ donor progenitors (Supplementary Figure S3) were present in the bone marrow at similar frequencies as wild-type when transplanted at a 1:1 ratio (Figure $3 \mathbf{A}$ ). Talin binds to the cytosolic tail of all integrin $\beta$ chains and is required for integrin activation (Kim et al., 2011). Similar to $\beta 1$ integrindeficient HoxB8-conditional progenitors, talin knockout $\left(T \ln 1^{-/-}\right)$progenitors exhibited significantly reduced engraftment compared to wild-type control (Figure 3A).

If transplanted donor HoxB8-conditional progenitors truly engraft and take up residence in the bone marrow niche, even if that residence is transient, one would expect to observe progenitor cell proliferation and progressive differentiation towards terminal leukocyte states. We have previously shown that cells in the bone marrow derived from transplanted HoxB8conditional progenitors that were loaded with a cell proliferation dye exhibit a greater than 20-fold dilution of the dye within 3 days, indicating in vivo proliferation of donor progenitors prior to their differentiation (Cohen et al., 2021). Analyses of all donorderived cells in the bone marrow at days 4 and 7 after transplant indicate that their composition shifts from being split between $\mathrm{cKit}^{+}{ }^{\mathrm{Ly}} 6 \mathrm{G}^{\text {low }}$ progenitors and $\mathrm{CKit}^{-} \mathrm{Ly}_{6 \mathrm{G}}{ }^{\text {high }}$ neutrophils at day 4 , 

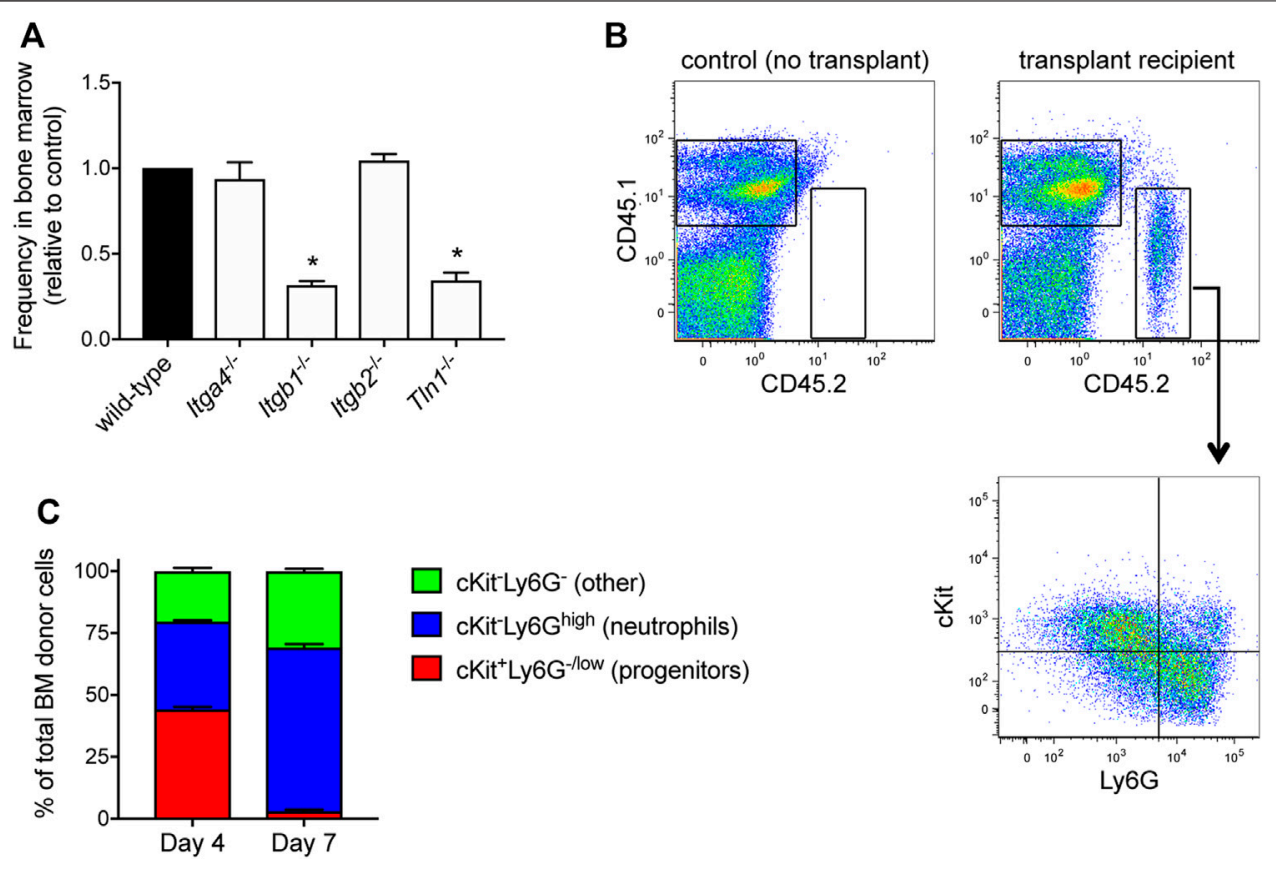

FIGURE 3 | HoxB8-conditional progenitor engraftment and in vivo differentiation. (A) Five days after CD45.1 mice received a 1:1 mix of wild-type and the indicated gene-deficient HoxB8-conditional progenitors, the relative frequency of wild-type and gene knockout cells among all donor-derived cells in the bone marrow was measured by flow cytometry. Data are presented as the relative frequency of each gene knockout type relative to its wild-type counter part, and normalized to the wildtype control (set to equal 1.0). Data were analyzed using one-way ANOVA. ${ }^{*} p<0.05$, compared to wild-type. (B) Example flow cytometry dotplots showing analyses of whole bone marrow from CD45.1 mice that received transplant of CD45.2 ${ }^{+}$HoxB8-conditional progenitors. Samples were labeled to determine expression of CD45.1, CD45.2, cKit and Ly6G. Gating for analyzing only donor-derived cells (top) and the subpopulations of donor-derived cells (bottom) in various states of maturity from progenitors/immature neutrophils (cKit ${ }^{+} \mathrm{L} 6 \mathrm{G}^{-1 \mathrm{ow}}$ ) towards terminal neutrophils (cKit ${ }^{-} \mathrm{Ly} 6 \mathrm{G}^{\text {high }}$ ). (C) Characterization and quantification of the in vivo differentiation of engrafted HoxB8-conditional progenitors at day 4 and day 7 after transplantation.

to predominantly $\mathrm{cKit}^{-} \mathrm{Ly}_{6 \mathrm{G}}{ }^{\text {high }}$ neutrophils at day 7 (Figures 3B,C). Taken together, these data suggest that HoxB8conditional progenitors home to the bone marrow and engraft in the hematopoietic niche, where they transiently proliferate and generate large numbers of mature neutrophils.

\section{Trafficking of Neutrophils Derived From Transplanted HoxB8-Conditional Progenitors}

Several studies have demonstrated that HoxB8-conditional progenitors can produce mature neutrophils following their transplantation into irradiated mice (Gautam et al., 2013; Redecke et al., 2013; Orosz et al., 2021). To further probe the dynamics of neutrophils generated in vivo from transplanted HoxB8-conditional progenitors, we first performed experiments using a model of sterile peritonitis induced by intraperitoneal injection of thiogylcollate. First, CD45.1 mice received transplantation of wild-type HoxB8-conditional progenitors and given sufficient time to produce robust chimerism in the blood. In response to peritonitis, we observed, as indicated by the similar levels of donor chimerism in the blood (before and after thioglycollate injection) and in the inflamed peritoneal cavity (Figure 4A). These data indicate that donor-derived neutrophils are recruited to a site of inflammation to an equal extent as endogenous host neutrophils.

Neutrophils mature in the bone marrow and their subsequent mobilization to the peripheral circulation is regulated by $\mathrm{C}-\mathrm{X}-\mathrm{C}$ chemokine receptor 2 (CXCR2) (Eash et al., 2010). To further understand whether neutrophils derived in vivo from transplanted progenitors are mobilized to the periphery and recruited to a site of inflammation via mechanisms that have been described for primary neutrophils, we performed several types of analyses in mice that had received a mix of wild-type and gene knockout donor progenitors. In these studies, CD45.1 mice received both wild-type and $\mathrm{C} x \mathrm{cr} 2^{-/-}$HoxB8-conditional progenitors and we restricted our analyses to $\mathrm{CD} 45.2^{+} \mathrm{Ly} 6 \mathrm{G}^{\text {high }}$ donor neutrophils (Supplementary Figure S4), as nearly 100\% of wild-type neutrophils (both host- and donor-derived) express CXCR2 (Supplementary Figure S5). At day 8 post-transplant, donor neutrophils of both genotypes were present in the bone marrow and peripheral blood of recipient mice. However, there was a significant reduction in the relative fraction of those donor neutrophils that were $\mathrm{Cxcr} 2^{-/-}$in the blood compared to the bone marrow (Figure 4B). These data indicate that homeostatic egress of mature donor-derived neutrophils to the periphery is regulated by CXCR2 in a similar manner to that of primary endogenous neutrophils (Eash et al., 2010). 

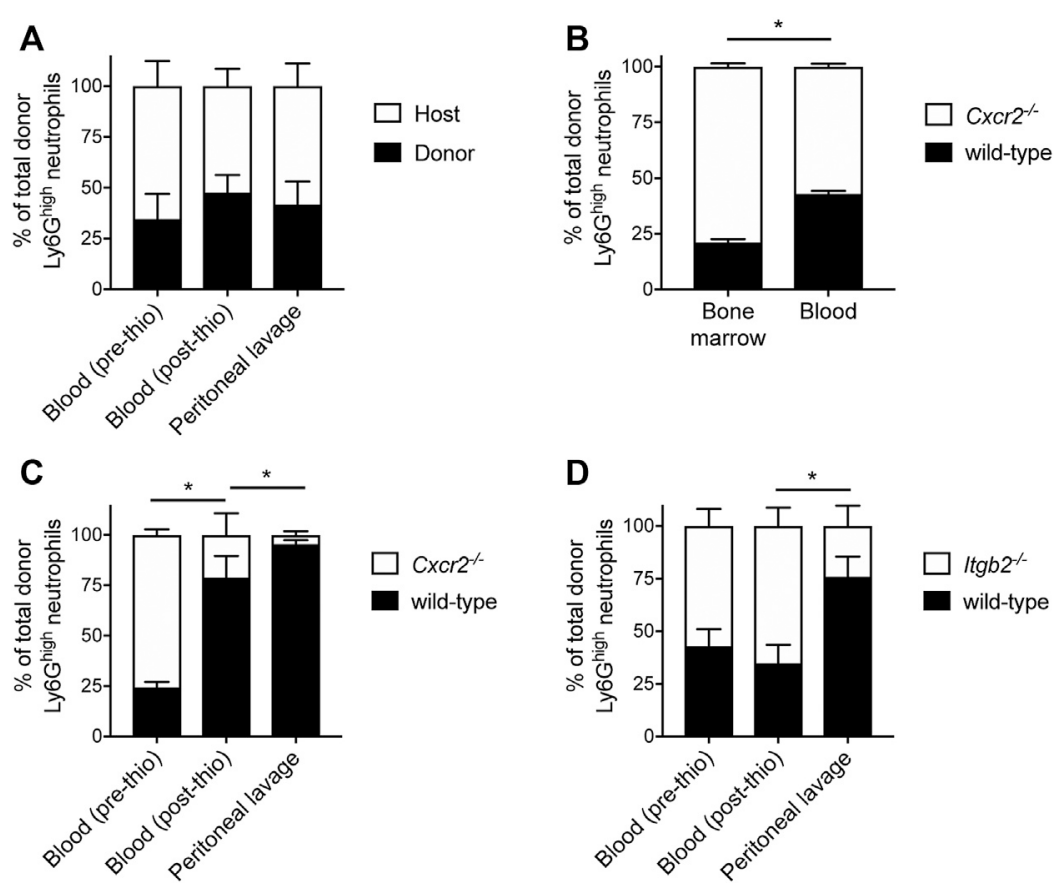

FIGURE 4 | Mechanisms underlying mobilization and recruitment of donor-derived neutrophils. Wild-type HoxB8-conditional progenitors, alone (A) or mixed with CXCR2-deficient (B,C) or 32 integrin-deficient HoxB8-conditional progenitors (D), were transplanted into CD45.1 recipient mice. (A, C,D) Mice were subjected to sterile peritonitis induced by thioglycollate. Blood samples (before and after thioglycollate injection) and peritoneal lavage were analyzed for the frequency of donor-derived wildtype and gene knockout neutrophils, as indicated. Data were analyzed using one-way ANOVA with Bonferroni post-hoc test for multiple comparisons. Statistical comparisons were made only between adjacent compartments/time points. ${ }^{*} p<0.05$. (B) In mice not subjected to any inflammatory stimulus, bone marrow and blood samples were collected at day 8 post-transplant and analyzed for the frequency of donor-derived wild-type and CXCR2-deficient neutrophils. Data were analyzed using a paired Student's t-test. ${ }^{*} p<0.05$.

To evaluate the role of CXCR2 in the mobilization of donor neutrophils in response to inflammatory stimuli, we analyzed the cellular composition of peripheral blood before and after mice received intraperitoneal thioglycollate to induce a sterile peritonitis. Similar to the analyses of homeostatic neutrophil egress to the periphery, the CD45.1 mice first received a transplant of a mix of CD45.2-expressing wild-type and $\mathrm{Cxcr}^{-/-}$HoxB8-conditional progenitors. The mix of donor progenitors for transplantation was composed of approximately one-third wild-type and two-thirds $C x \mathrm{Cr}^{-/-}$ progenitors, as we wanted to be able to detect changes in donor neutrophil fraction with the expectation that CXCR2 is involved both in neutrophil mobilization and recruitment, as is known for primary murine neutrophils (Cacalano et al., 1994; Eash et al., 2010). At $90 \mathrm{~min}$ after inducing inflammation, there was a significant decrease in the fraction of donor-derived CXCR2-deficient neutrophils in the blood (Figure 4C), indicating a CXCR2-dependent mobilization of donor neutrophils from the bone marrow. There was a further reduction in the fraction of donor-derived $\mathrm{Cxcr2} 2^{-/-}$ neutrophils relative to wild-type in the peritoneal lavage, relative to the blood (Figure 4C). Taken together, these data confirm that both the stimulated mobilization and recruitment of neutrophils generated in vivo from transplanted HoxB8conditional progenitors occurs via CXCR2-dependent mechanisms.
Endogenous host neutrophils and those derived in vivo from transplanted donor HoxB8-conditional progenitors express similar levels of adhesion and signaling receptors involved in regulating neutrophil recruitment, including PSGL-1, L-selectin, CD11a, CD11b, CXCR2, and Fc $\gamma$ RIV (Supplementary Figure S5). These data are consistent with a recent study, with the exception that we observed equal expression of CD11b and Fc $\gamma$ RIV on donor and host neutrophils (Supplementary Figure S5), whereas those receptors were elevated on neutrophils when produced in irradiated recipient mice (Orosz et al., 2021). Two of the major types of $\beta 2$ integrins expressed by neutrophils, LFA-1 ( $\alpha \mathrm{L} \beta 2$ ) and Mac-1 ( $\alpha \mathrm{M} \beta 2)$, play key roles in neutrophil recruitment by mediating adhesion to inflamed endothelium (Ley et al., 2007). To understand whether donorderived neutrophils are recruited to sites of inflammation via adhesion mechanisms that have been well described for hostderived primary murine neutrophils, we again used a model of thioglycollate-induced peritonitis in mice that received a 1:1 mix of wild-type and $\beta 2$ integrin-deficient $\left(\operatorname{Itgb2} 2^{-/-}\right.$) HoxB8conditional progenitors. While there was a similar proportion of wild-type and Itgb2 ${ }^{-/-}$donor neutrophils in the blood both before and after inducing inflammation, we observed a significant decrease in the fraction of $\beta 2$ integrin-deficient donor-derived neutrophils in the peritoneal lavage (Figure 4D). These data indicate that, like primary host neutrophils, neutrophils derived in vivo from transplanted HoxB8-conditional progenitors are 

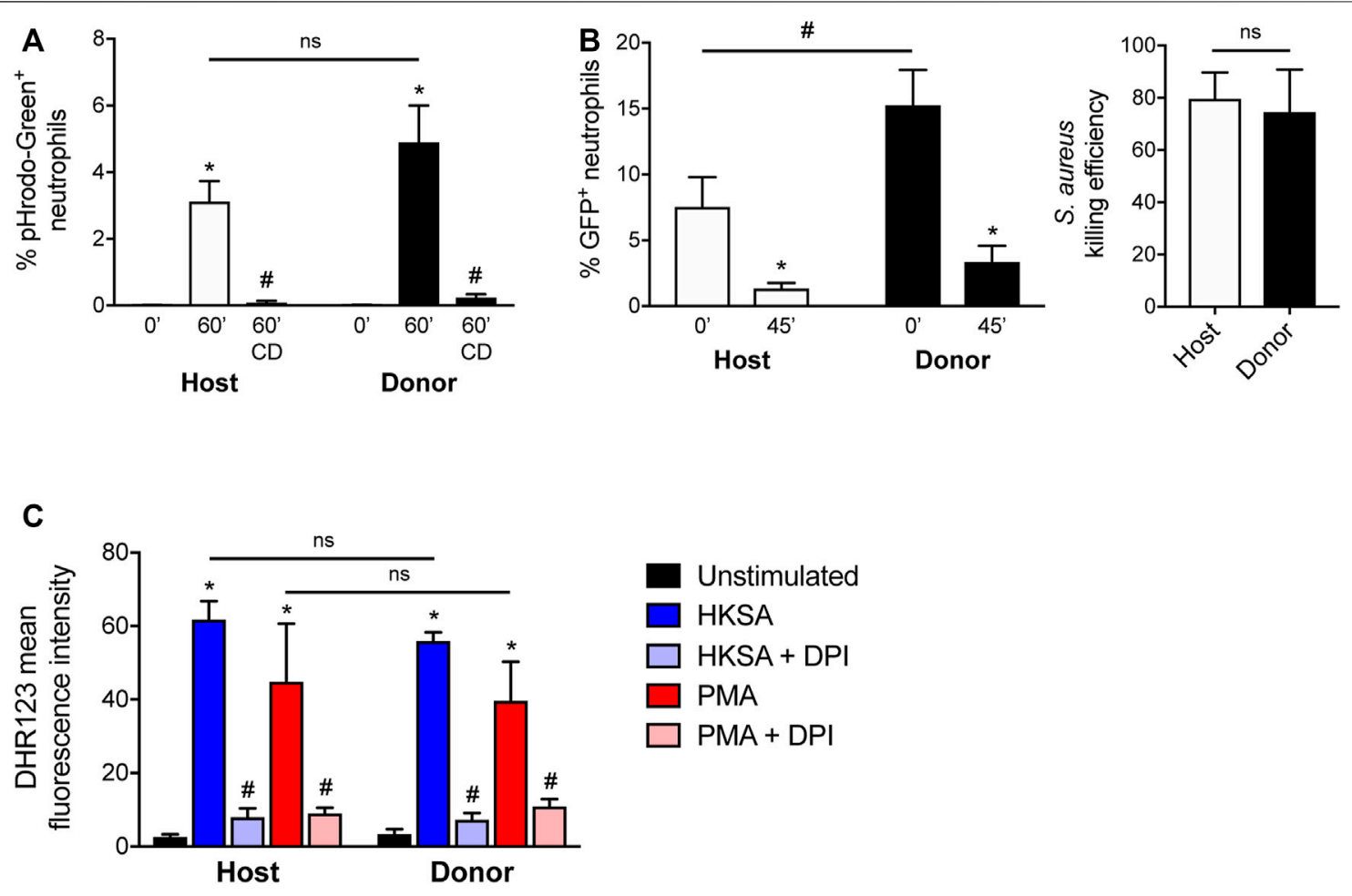

FIGURE 5 | Effector function of neutrophils derived in vivo from HoxB8-conditional progenitors. Seven days after CD45.1 mice received transplant of wild-type HoxB8-conditional progenitors, blood samples were subjected to assays of neutrophil function using flow cytometry. CD45.2 ${ }^{+}{\text {Ly } 6 G^{+}}$donor-derived neutrophils were distinguished from CD45.1 ${ }^{+}$Ly6G ${ }^{+}$host-derived neutrophils for comparative analyses. (A) Host and donor neutrophil phagocytosis of pHrodo-Green conjugated S. aureus, measured after 60 min exposure in the absence or presence of $10 \mu \mathrm{g} / \mathrm{ml}$ cytochalasin D (CD). Data were analyzed using two-way ANOVA with Bonferroni post-hoc test for multiple comparisons. ${ }^{*} p<0.05$, compared to 0 min $\# p<0.05$, compared to 60 min. (B) Host and donor neutrophil intracellular killing of $S$. aureus, as measured by the fraction of neutrophils remaining GFP ${ }^{+} 45$ min after allowing initial phagocytosis of strain USA300-sGFP. The killing efficiency was calculated by comparing the end point fraction of $\mathrm{GFP}^{+}$neutrophils to the starting point (0 min). Data were analyzed using two-way ANOVA with Bonferroni post-hoc test for multiple comparisons. * $p<0.05$, compared to 0 min \#p < 0.05, as indicated. Killing efficiency data were analyzed by an unpaired Student's t-test. (C) Quantification of stimulated ROS generation by host and donor neutrophils, expressed as the mean fluorescence intensity of DHR123. Blood samples were stimulated with either heat-killed $S$. aureus (HKSA) or $100 \mathrm{ng} / \mathrm{ml}$ PMA, in the absence or presence of $10 \mu \mathrm{M}$ DPI. Data were analyzed using two-way ANOVA with Bonferroni post-hoc test for multiple comparisons. ${ }^{*} p<0.05$, compared to unstimulated. $\# p<0.05$, compared to HKSA or PMA alone.

recruited to the inflamed peritoneum via $\beta 2$ integrin-dependent mechanisms.

\section{Effector Functions of Donor Neutrophils Derived in vivo}

Previous studies have characterized the effector functions of mature neutrophils derived in vitro and in vivo from HoxB8conditional progenitors (McDonald et al., 2011; Weiss et al., 2018; Zehrer et al., 2018; Chu et al., 2019; Saul et al., 2019; Orosz et al., 2021). Some of these studies are limited to evaluating only the function of neutrophils derived from HoxB8-conditional progenitors in vitro or lack a controlled in vivo comparison with endogenous host primary neutrophils. Since the unique line of HoxB8-conditional progenitors that we describe in this study engrafts in unconditioned mice, we were therefore able to directly assay both host and donor neutrophil functions under internally controlled conditions. Using CD45.1 mice that received CD $45.2^{+}$donor progenitors, we assayed neutrophil effector functions using blood samples. First, we exposed blood samples to Staphylococcus aureus bioparticles conjugated to a pHrodo-Green dye that fluoresces upon internalization into the neutrophil phagolysosome, using a low particle:cell ratio so as to better resolve potential differences between host- and donorderived neutrophils (Supplementary Figure S6). We observed that neutrophils derived in vivo from transplanted HoxB8conditional progenitors had a similar capacity for $S$. aureus bioparticle phagocytosis that was inhibited by the actindisrupting agent cytochalasin D (Figure 5A).

To determine whether neutrophils are able to kill $S$. aureus after uptake, we employed an assay based upon the intracellular killing event being shown to result in the bleaching of GFP expressed by a strain of S. aureus (Schwartz et al., 2009). Donor-derived blood neutrophils were able to eradicate live $S$. aureus to a similar extent as host-derived neutrophils (Figure 5B). Neutrophil intracellular killing of $S$. aureus occurs in a manner dependent on the NADPH oxidase (NOX2). Both host- and donor-derived neutrophils underwent NOX2-mediated respiratory burst in response to heat-killed $S$. aureus or PMA, and sensitive to the NOX2 inhibitor DPI 
(Figure 5C). Together, these data indicate that neutrophils generated from HoxB8-conditional progenitors following their transplantation into naïve mice have competent host defense effector functions.

\section{DISCUSSION}

Neutrophils are essential players in the acute inflammatory response. In recent years, investigators have continued to identify novel and diverse roles for neutrophils in many facets of host defense and immune regulation (Kubes, 2018). While neutrophil biologists have long faced experimental limitations and challenges, the emergence of the HoxB8-conditional progenitor system has provided a means for researchers to obtain near-primary neutrophils that are genetically tractable prior to their differentiation. Here, we report the discovery of a unique derivation of a HoxB8-conditional progenitor cell line that is capable of engrafting in naive mice and results in robust donor neutrophil chimerism that lasts for more than a week.

Hematopoietic stem cell transplantation (HSCT) can be curative for blood cell disorders and malignancies. HSPCs reside in specialized niches within the extravascular space of the bone marrow. For HSCT to be successful, transplanted HSPCs must traffic to these specialized hematopoietic niches and then take up residence through a process called engraftment. It remains a topic of debate whether the engraftment of transplanted HSPCs is limited by the occupancy of specialized niches by host HSPCs (Bhattacharya et al., 2008). Clinically, HSCT recipients undergo irradiation- and/or chemical-induced ablation of the hematopoietic system prior to the injection of HSCs or whole bone marrow. This conditioning of the host promotes donor engraftment through a combination of blocking rejection of the graft by the host immune response and freeing up hematopoietic niche space for donor HSPCs. In the absence of conditioning, while HSC engraftment remains poor even in recipients that are incapable of graft rejection (Muller et al., 2000; Czechowicz et al., 2007; Bhattacharya et al., 2008), the availability of niches for specific subsets of multipotent and lineage-restricted progenitors has not been extensively investigated. However, our derivation of a HoxB8-conditional progenitor cell line that efficiently engrafts in unconditioned mice appears to be a novel discovery, as we found that other independently generated HoxB8-conditional progenitor lines did not engraft and others have demonstrated the absolute requirement for irradiating recipient mice in order to achieve engraftment and donor neutrophil chimerism (Orosz et al., 2021).

Similar to the investigation of HSPCs and conditioning the hematopoietic niche, the mechanisms of HSC homing and engraftment are more clearly defined than those of progenitor subsets with restricted multipotency. The integrin VLA-4 ( $\alpha 4 \beta 1)$ is essential for the homing of transplanted HSCs to the bone marrow (Papayannopoulou et al., 1995; Potocnik et al., 2000; Qian et al., 2007; Rettig et al., 2012). We were therefore surprised to observe that Itga4 ${ }^{-1-}$ HoxB8-conditional progenitors lacking VLA-4 expression engrafted and proliferated in the bone marrow of recipient mice as well as wild-type progenitors. By contrast, $\beta 1$ integrin-deficient HoxB8-conditional progenitors have impaired homing and/or engraftment, suggesting that a $\beta 1$ integrin subtype other than VLA-4 plays a role in this process. Furthermore, since talin knockout HoxB8-conditional progenitors were also reduced in their bone marrow frequency relative to wild-type, it would suggest a homing mechanism that involves activated $\beta 1$ integrins, similar to that observed for $\beta 2$ integrins and neutrophil trafficking (Lefort et al., 2012). Further investigation of the mechanisms of engraftment for HoxB8conditional progenitors will also provide insight as to how this cell line is uniquely able to engraft in the absence of myeloablation.

In recent studies, single-cell technologies have enabled the identification of the various neutrophil precursor states that define the maturation process following lineage commitment (Evrard et al., 2018; Zhu et al., 2018; Kwok et al., 2020). Our analyses of cell surface receptors and markers reveal that the progenitor identity that is conditionally immortalized through enforced HoxB8 expression appears to vary between several independent derivations, despite following the same protocol. HoxB8-conditional progenitor line 1 that we describe in this study may be best characterized as a granulocyte macrophage progenitor (GMP), given the bifurcated expression of Ly6C by the parental progenitor line 1 and single-cell clones derived from it, a pattern that exists within GMPs and may be related to lineage commitment decisions (Kwok et al., 2020). Comparing the timing of in vivo differentiation in our studies and those recently reported for neutrophil-committed preNeus (Evrard et al., 2018), the HoxB8-conditional progenitor lines we characterize are unlikely to be in the preNeu stage while HoxB8 is expressed since we did not observe a substantial shift towards $\mathrm{CKit}^{-}{ }^{\mathrm{Ly}} 6 \mathrm{G}^{\text {high }}$ donor cells in the bone marrow until day 4 and later. Future systematic analyses are necessary to understand the divergent outcomes of HoxB8-dependent progenitor immortalization with respect to their developmental identity and eventual engraftment potential as an established cell line.

Our studies confirm that neutrophils derived in vivo from donor progenitors are biologically comparable to endogenous neutrophils, building upon the results of others that have utilized transplantation of HoxB8-conditional progenitors into mice (Gautam et al., 2013; Orosz et al., 2021). In order to show this more definitively, the experiments described here are, to our knowledge, the first to extensively probe the molecular mechanisms of trafficking and function of neutrophils generated in vivo from transplanted HoxB8-conditional progenitors. From CXCR2-dependent mobilization from the bone marrow to neutrophil recruitment mediated by CXCR2 and $\beta 2$ integrins, we demonstrate that donor-derived neutrophils are functionally equivalent to endogenous host neutrophils. We found that HoxB8-conditional progenitor differentiation that occurs in vivo in the microenvironment of the bone marrow allows donor neutrophils to reach their full functional competence. This contrasts with certain neutrophil functions observed with in vitro differentiation of HoxB8conditional progenitors, under conditions that are unlikely to fully recapitulate the environment in which neutrophil maturation occurs in the bone marrow. For example, NOX2-dependent stimulated 
generation of ROS is more than an order of magnitude lower for in vitro-differentiated neutrophils compared to primary neutrophils (Saul et al., 2019). Our results show that ROS generation in response to either $S$. aureus bioparticles or PMA is no different between endogenous host neutrophils and those derived in vivo from transplanted HoxB8-conditional progenitors. In addition, intracellular eradication of $S$. aureus occurs as well in donorderived neutrophils as in host-derived neutrophils. Taken together, our results strongly suggest that mature neutrophils that are generated in mice from adoptively transplanted HoxB8conditional progenitors are biologically equivalent to primary neutrophils.

Multidrug-resistant (MDR) bacterial and fungal infections are an increasingly urgent clinical challenge, especially for opportunistic pathogens that cause severe disease in the immunocompromised. Due to a number of factors, the pipeline for new antibiotics has seen a drastic reduction in productivity (Lewis, 2013). Immunomodulatory and cell therapeutic strategies are being pursued as a complementary approach or alternative to antibiotics (Hancock et al., 2012; Kumaresan et al., 2014). While granulocyte transfusion therapy has experienced a long and detoured path in clinical implementation (Marfin and Price, 2015), it may be worth revisiting its conceptual basis as new approaches are developed for generating neutrophils (both ex vivo and in vivo). We posit that conditionally-immortalized neutrophil progenitors could be an effective cellular therapeutic in the context of neutropenia or a dysregulated inflammatory response. Given their genetic tractability, murine HoxB8-conditional progenitors represent an innovative system for investigating means to enhance neutrophil effector functions. In this way, an off-the-shelf product that transiently generates neutrophils with superior capacity to, for example, overcome evasive intracellular survival of MDR S. aureus (Greenlee-Wacker et al., 2015) may be an option alongside antibiotics of last resort.

There remain some limitations to the study reported here. The technical aspects in deriving HoxB8-conditional progenitors that lead to cell lines with high engraftment capacity in unconditioned mice, as compared those that lack engraftment capacity that have been demonstrated here and by others (Orosz et al., 2021), remain unclear and should be the subject of future studies. We posit that the timing of HoxB8 induction in HSPCs relative to their isolation may influence the progenitor type that is conditionally-immortalized. For our experiments, recipient mice exhibited donor neutrophil chimerism in the periphery that took 4 days to reached levels above $10 \%$, suggesting limitations to the potential therapeutic applications of neutrophil progenitors. Further investigation into modulating the kinetics of in vivo proliferation and/or differentiation may broaden the translational impact in this new area of cell therapies to augment host defense. Future studies are also needed to understand whether neutrophils generated in vivo from transplanted progenitors exhibit subsets with transcriptional heterogeneity, as has been described for endogenous bone marrow and blood neutrophils in the mouse (Xie et al., 2020).

In conclusion, our studies describe a new HoxB8-conditional progenitor cell line that is uniquely capable of transient engraftment in the unconditioned murine host in a manner that depends on $\beta 1$ integrins, but not VLA-4. Within the bone marrow, transplanted HoxB8-conditional progenitors proliferate and differentiate into mature neutrophils that are mechanistically and functionally the equivalent of primary neutrophils. These results further confirm the utility of the HoxB8 progenitor system for experimental neutrophil biology and suggest future translational avenues.

\section{DATA AVAILABILITY STATEMENT}

The raw data supporting the conclusion of this article will be made available by the authors, without undue reservation.

\section{ETHICS STATEMENT}

The animal study was reviewed and approved by Lifespan Animal Welfare Committee, Rhode Island Hospital.

\section{AUTHOR CONTRIBUTIONS}

JC: Developing cell lines, designing research studies, conducting experiments, analyzing data. MD: Developing cell lines, designing research studies, conducting experiments, analyzing data. $\mathrm{KH}$ : Designing research studies, conducting experiments, analyzing data. BN: Designing research studies, conducting experiments, analyzing data. RJ: Designing research studies, conducting experiments. ZW: Developing cell lines. AC: Designing research studies, analyzing data. PD: Designing research studies. CL: Supervising the project, designing research studies, conducting experiments, analyzing data, writing the manuscript.

\section{FUNDING}

This research was supported by awards from the National Institutes of Health (GM124911 to CL; GM119943 and CA218079 to PD; T32GM065085 supported JC; and T32HL134625 supported BN), Brown Physicians, Inc. (CL), and the Armand D. Versaci Research Scholarship in Surgical Sciences (MD).

\section{ACKNOWLEDGMENTS}

We thank Dr. Paul Ekert, Dr. Patrice Dubreuil, and Dr. Alexander Horswill for kindly providing reagents/resources.

\section{SUPPLEMENTARY MATERIAL}

The Supplementary Material for this article can be found online at: https://www.frontiersin.org/articles/10.3389/fcell.2022.840894/ full\#supplementary-material 


\section{REFERENCES}

Bhattacharya, D., Ehrlich, L. I. R., and Weissman, I. L. (2008). Space-time Considerations for Hematopoietic Stem Cell Transplantation. Eur. J. Immunol. 38 (8), 2060-2067. doi:10.1002/eji.200838383

Blanter, M., Gouwy, M., and Struyf, S. (2021). Studying Neutrophil Function In Vitro: Cell Models and Environmental Factors. Jir 14, 141-162. doi:10.2147/JIR. S284941

Boztug, K., Appaswamy, G., Ashikov, A., Schäffer, A. A., Salzer, U., Diestelhorst, J., et al. (2009). A Syndrome with Congenital Neutropenia and Mutations inG6PC3. N. Engl. J. Med. 360 (1), 32-43. doi:10.1056/NEJMoa0805051

Cacalano, G., Lee, J., Kikly, K., Ryan, A. M., Pitts-Meek, S., Hultgren, B., et al. (1994). Neutrophil and B Cell Expansion in Mice that Lack the Murine IL-8 Receptor Homolog. Science 265 (5172), 682-684. doi:10.1126/science.8036519

Cheung, Y. Y., Kim, S. Y., Yiu, W. H., Pan, C.-J., Jun, H.-S., Ruef, R. A., et al. (2007). Impaired Neutrophil Activity and Increased Susceptibility to Bacterial Infection in Mice Lacking Glucose-6-Phosphatase-ß. J. Clin. Invest. 117 (3), 784-793. doi:10.1172/JCI30443

Chu, J. Y., McCormick, B., Mazelyte, G., Michael, M., and Vermeren, S. (2019). HoxB8 Neutrophils Replicate Fc $\gamma$ Receptor and Integrin-induced Neutrophil Signaling and Functions. J. Leukoc. Biol. 105 (1), 93-100. doi:10.1002/JLB. 1AB0618-232R

Cohen, J. T., Danise, M., Machan, J. T., Zhao, R., and Lefort, C. T. (2021). Murine Myeloid Progenitors Attenuate Immune Dysfunction Induced by Hemorrhagic Shock. Stem Cel Rep. 16 (2), 324-336. doi:10.1016/j.stemcr.2020.12.014

Collins, S. J., Ruscetti, F. W., Gallagher, R. E., and Gallo, R. C. (1978). Terminal Differentiation of Human Promyelocytic Leukemia Cells Induced by Dimethyl Sulfoxide and Other Polar Compounds. Proc. Natl. Acad. Sci. 75 (5), 2458-2462. doi:10.1073/pnas.75.5.2458

Czechowicz, A., Kraft, D., Weissman, I. L., and Bhattacharya, D. (2007). Efficient Transplantation via Antibody-Based Clearance of Hematopoietic Stem Cell Niches. Science 318 (5854), 1296-1299. doi:10.1126/science.1149726

Duran-Struuck, R., and Dysko, R. C. (2009). Principles of Bone Marrow Transplantation (BMT): Providing Optimal Veterinary and Husbandry Care to Irradiated Mice in BMT Studies. J. Am. Assoc. Lab. Anim. Sci. 48 (1), 11-22.

Eash, K. J., Greenbaum, A. M., Gopalan, P. K., and Link, D. C. (2010). CXCR2 and CXCR4 Antagonistically Regulate Neutrophil Trafficking from Murine Bone Marrow. J. Clin. Invest. 120 (7), 2423-2431. doi:10.1172/JCI41649

Evrard, M., Kwok, I. W. H., Chong, S. Z., Teng, K. W. W., Becht, E., Chen, J., et al. (2018). Developmental Analysis of Bone Marrow Neutrophils Reveals Populations Specialized in Expansion, Trafficking, and Effector Functions. Immunity 48 (2), 364-379. doi:10.1016/j.immuni.2018.02.002

Gautam, S., Kirschnek, S., Gentle, I. E., Kopiniok, C., Henneke, P., Häcker, H., et al. (2013). Survival and Differentiation Defects Contribute to Neutropenia in Glucose-6-Phosphatase- $\beta$ (G6PC3) Deficiency in a Model of Mouse Neutrophil Granulocyte Differentiation. Cell Death Differ 20 (8), 1068-1079. doi:10.1038/cdd.2013.39

Greenlee-Wacker, M., DeLeo, F. R., and Nauseef, W. M. (2015). How MethicillinResistant Staphylococcus aureus Evade Neutrophil Killing. Curr. Opin. Hematol. 22 (1), 30-35. doi:10.1097/MOH.0000000000000096

Gupta, D., Shah, H. P., Malu, K., Berliner, N., and Gaines, P. (2014). Differentiation and Characterization of Myeloid Cells. Curr. Protoc. Immunol. 104, Unit 22F 25. doi:10.1002/0471142735.im22f05s104

Hancock, R. E. W., Nijnik, A., and Philpott, D. J. (2012). Modulating Immunity as a Therapy for Bacterial Infections. Nat. Rev. Microbiol. 10 (4), 243-254. doi:10. 1038/nrmicro2745

Kim, C., Ye, F., and Ginsberg, M. H. (2011). Regulation of Integrin Activation. Annu. Rev. Cel Dev. Biol. 27, 321-345. doi:10.1146/annurev-cellbio-100109104104

Knoepfler, P. S., Sykes, D. B., Pasillas, M., and Kamps, M. P. (2001). HoxB8 Requires its Pbx-Interaction Motif to Block Differentiation of Primary Myeloid Progenitors and of Most Cell Line Models of Myeloid Differentiation. Oncogene 20 (39), 5440-5448. doi:10.1038/sj.onc.1204710

Krishnaraju, K., Hoffman, B., and Liebermann, D. A. (1997). Lineage-specific Regulation of Hematopoiesis by HOX-B8 (HOX-2.4): Inhibition of Granulocytic Differentiation and Potentiation of Monocytic Differentiation. Blood 90 (5), 1840-1849. doi:10.1182/blood.v90.5.1840
Kubes, P. (2018). The Enigmatic Neutrophil: what We Do Not Know. Cell Tissue Res 371 (3), 399-406. doi:10.1007/s00441-018-2790-5

Kumaresan, P. R., Manuri, P. R., Albert, N. D., Maiti, S., Singh, H., Mi, T., et al. (2014). Bioengineering T Cells to Target Carbohydrate to Treat Opportunistic Fungal Infection. Proc. Natl. Acad. Sci. 111 (29), 10660-10665. doi:10.1073/ pnas.1312789111

Kwok, I., Becht, E., Xia, Y., Ng, M., Teh, Y. C., Tan, L., et al. (2020). Combinatorial Single-Cell Analyses of Granulocyte-Monocyte Progenitor Heterogeneity Reveals an Early Uni-Potent Neutrophil Progenitor. Immunity 53 (2), 303-318. doi:10.1016/j.immuni.2020.06.005

Lawson, N. D., Krause, D. S., and Berliner, N. (1998). Normal Neutrophil Differentiation and Secondary Granule Gene Expression in the EML and MPRO Cell Lines. Exp. Hematol. 26 (12), 1178-1185.

Lefort, C. T., Rossaint, J., Moser, M., Petrich, B. G., Zarbock, A., Monkley, S. J., et al. (2012). Distinct Roles for Talin-1 and Kindlin-3 in LFA-1 Extension and Affinity Regulation. Blood 119 (18), 4275-4282. doi:10.1182/blood-2011-08373118

Lewis, K. (2013). Platforms for Antibiotic Discovery. Nat. Rev. Drug Discov. 12 (5), 371-387. doi:10.1038/nrd3975

Ley, K., Hoffman, H. M., Kubes, P., Cassatella, M. A., Zychlinsky, A., Hedrick, C. C., et al. (2018). Neutrophils: New Insights and Open Questions. Sci. Immunol. 3 (30). doi:10.1126/sciimmunol.aat4579

Ley, K., Laudanna, C., Cybulsky, M. I., and Nourshargh, S. (2007). Getting to the Site of Inflammation: the Leukocyte Adhesion cascade Updated. Nat. Rev. Immunol. 7 (9), 678-689. doi:10.1038/nri2156

Marfin, A. A., and Price, T. H. (2015). Granulocyte Transfusion Therapy. J. Intensive Care Med. 30 (2), 79-88. doi:10.1177/0885066613498045

McDonald, J. U., Cortini, A., Rosas, M., Fossati-Jimack, L., Ling, G. S., Lewis, K. J., et al. (2011). In Vivo functional Analysis and Genetic Modification of In Vitro -derived Mouse Neutrophils. FASEB j. 25 (6), 1972-1982. doi:10.1096/fj.10178517

Mei, Y., Han, X., Liu, Y., Yang, J., Sumagin, R., and Ji, P. (2020). Diaphanousrelated Formin mDia2 Regulates Beta2 Integrins to Control Hematopoietic Stem and Progenitor Cell Engraftment. Nat. Commun. 11 (1), 3172. doi:10. 1038/s41467-020-16911-4

Müller, S. M., Kohn, T., Schulz, A. S., Debatin, K. M., and Friedrich, W. (2000). Similar Pattern of Thymic-dependent T-Cell Reconstitution in Infants with Severe Combined Immunodeficiency after Human Leukocyte Antigen (HLA)identical and HLA-Nonidentical Stem Cell Transplantation. Blood 96 (13), 4344-4349.

Orosz, A., Walzog, B., and Mócsai, A. (2021). In Vivo Functions of Mouse Neutrophils Derived from HoxB8-Transduced Conditionally Immortalized Myeloid Progenitors. J.I. 206 (2), 432-445. doi:10.4049/jimmunol.2000807

Papayannopoulou, T., Craddock, C., Nakamoto, B., Priestley, G. V., and Wolf, N. S. (1995). The VLA4/VCAM-1 Adhesion Pathway Defines Contrasting Mechanisms of Lodgement of Transplanted Murine Hemopoietic Progenitors between Bone Marrow and Spleen. Proc. Natl. Acad. Sci. 92 (21), 9647-9651. doi:10.1073/pnas.92.21.9647

Potocnik, A. J., Brakebusch, C., and Fässler, R. (2000). Fetal and Adult Hematopoietic Stem Cells Require $\beta 1$ Integrin Function for Colonizing Fetal Liver, Spleen, and Bone Marrow. Immunity 12 (6), 653-663. doi:10.1016/s10747613(00)80216-2

Qian, H., Georges-Labouesse, E., Nyström, A., Domogatskaya, A., Tryggvason, K., Jacobsen, S. E. W., et al. (2007). Distinct Roles of Integrins a6 and $\alpha 4$ in Homing of Fetal Liver Hematopoietic Stem and Progenitor Cells. Blood 110 (7), 2399-2407. doi:10.1182/blood-2006-10-051276

Redecke, V., Wu, R., Zhou, J., Finkelstein, D., Chaturvedi, V., High, A. A., et al. (2013). Hematopoietic Progenitor Cell Lines with Myeloid and Lymphoid Potential. Nat. Methods 10 (8), 795-803. doi:10.1038/nmeth.2510

Rettig, M. P., Ansstas, G., and DiPersio, J. F. (2012). Mobilization of Hematopoietic Stem and Progenitor Cells Using Inhibitors of CXCR4 and VLA-4. Leukemia 26 (1), 34-53. doi:10.1038/leu.2011.197

Salmanidis, M., Brumatti, G., Narayan, N., Green, B. D., van den Bergen, J. A., Sandow, J. J., et al. (2013). Hoxb8 Regulates Expression of microRNAs to Control Cell Death and Differentiation. Cel Death Differ 20 (10), 1370-1380. doi:10.1038/cdd.2013.92

Saul, S., Castelbou, C., Fickentscher, C., and Demaurex, N. (2019). Signaling and Functional Competency of Neutrophils Derived from Bone-marrow Cells 
Expressing the ER-HOXB8 Oncoprotein. J. Leukoc. Biol. 106 (5), 1101-1115. doi:10.1002/JLB.2A0818-314R

Schwartz, J., Leidal, K. G., Femling, J. K., Weiss, J. P., and Nauseef, W. M. (2009). Neutrophil Bleaching of GFP-Expressing Staphylococci: Probing the Intraphagosomal Fate of Individual Bacteria. J. Immunol. 183 (4), 2632-2641. doi:10.4049/jimmunol.0804110

Valtieri, M., Tweardy, D. J., Caracciolo, D., Johnson, K., Mavilio, F., Altmann, S., et al. (1987). Cytokine-dependent Granulocytic Differentiation. Regulation of Proliferative and Differentiative Responses in a Murine Progenitor Cell Line. J. Immunol. 138 (11), 3829-3835.

Wang, G. G., Calvo, K. R., Pasillas, M. P., Sykes, D. B., Häcker, H., and Kamps, M. P. (2006). Quantitative Production of Macrophages or Neutrophils Ex Vivo Using Conditional Hoxb8. Nat. Methods 3 (4), 287-293. doi:10.1038/nmeth865

Weiss, E., Hanzelmann, D., Fehlhaber, B., Klos, A., Loewenich, F. D., Liese, J., et al. (2018). Formyl-peptide Receptor 2 Governs Leukocyte Influx in Local Staphylococcus aureus Infections. FASEB j. 32 (1), 26-36. doi:10.1096/f..201700441R

Wilson, Z. S., Ahn, L. B., Serratelli, W. S., Belley, M. D., Lomas-Neira, J., Sen, M., et al. (2017). Activated $\beta 2$ Integrins Restrict Neutrophil Recruitment during Murine Acute Pseudomonal Pneumonia. Am. J. Respir. Cel Mol Biol 56 (5), 620-627. doi:10.1165/rcmb.2016-0215OC

Xie, X., Shi, Q., Wu, P., Zhang, X., Kambara, H., Su, J., et al. (2020). Single-cell Transcriptome Profiling Reveals Neutrophil Heterogeneity in Homeostasis and Infection. Nat. Immunol. 21 (9), 1119-1133. doi:10.1038/s41590-020-0736-z

Xun, C., Thompson, J., Jennings, C., Brown, S., and Widmer, M. (1994). Effect of Total Body Irradiation, Busulfan-Cyclophosphamide, or Cyclophosphamide Conditioning on Inflammatory Cytokine Release and Development of Acute and Chronic Graft-Versus-Host Disease in H-2- Incompatible Transplanted
SCID Mice. Blood 83 (8), 2360-2367. doi:10.1182/blood.v83.8.2360. bloodjournal 8382360

Zehrer, A., Pick, R., Salvermoser, M., Boda, A., Miller, M., Stark, K., et al. (2018). A Fundamental Role of Myh9 for Neutrophil Migration in Innate Immunity. J.I. 201 (6), 1748-1764. doi:10.4049/jimmunol.1701400

Zhu, Y. P., Padgett, L., Dinh, H. Q., Marcovecchio, P., Blatchley, A., Wu, R., et al. (2018). Identification of an Early Unipotent Neutrophil Progenitor with Protumoral Activity in Mouse and Human Bone Marrow. Cel Rep. 24 (9), 2329-2341. doi:10.1016/j.celrep.2018.07.097

Conflict of Interest: The authors declare that the research was conducted in the absence of any commercial or financial relationships that could be construed as a potential conflict of interest.

Publisher's Note: All claims expressed in this article are solely those of the authors and do not necessarily represent those of their affiliated organizations, or those of the publisher, the editors, and the reviewers. Any product that may be evaluated in this article, or claim that may be made by its manufacturer, is not guaranteed or endorsed by the publisher.

Copyright (C) 2022 Cohen, Danise, Hinman, Neumann, Johnson, Wilson, Chorzalska, Dubielecka and Lefort. This is an open-access article distributed under the terms of the Creative Commons Attribution License (CC BY). The use, distribution or reproduction in other forums is permitted, provided the original author(s) and the copyright owner(s) are credited and that the original publication in this journal is cited, in accordance with accepted academic practice. No use, distribution or reproduction is permitted which does not comply with these terms. 